AIAA-80-0135

\title{
A TWO-LAYER CALCULATION FOR THE INITIAL INTERACTION REGION OF AN UNSEPARATED SUPERSONIC TURBULENT BOUNDARY LAYER WITH A RAMP
}

\author{
R. ROSEN, A. ROSHKO, AND D. L. PAVISH \\ McDONNEL DOUGLAS ASTRONAUTICS COMPANY \\ HUNTINGTON BEACH, CALIFORNIA
}

\section{AIAA 18th AEROSPACE SCIENCES MEETING January 14-16, 1980/Pasadena, California}




\author{
R. Rosen*, A. Roshko**, and D. L. Pavish ${ }^{\dagger}$ \\ McDonnell Douglas Astronautics Company \\ Huntington Beach, California
}

\begin{abstract}
The interaction of an unseparated supersonic turbulent boundary layer with a compression corner produces an extremely rapid rise in pressure at the corner, followed by a more gradual increase to the final pressure. In this paper, the flow in the corner region is analyzed by an integral method with the objective of predicting the initial pressure rise. Comparisons with experimental pressure rise data are presented for cases covering supersonic and hypersonic flows of practical interest. Also presented are some calculations and comparisons of downstream pressure distributions obtained by using the predicted corner rise as the first point in an existing inviscid method.

\section{Nomenclature}

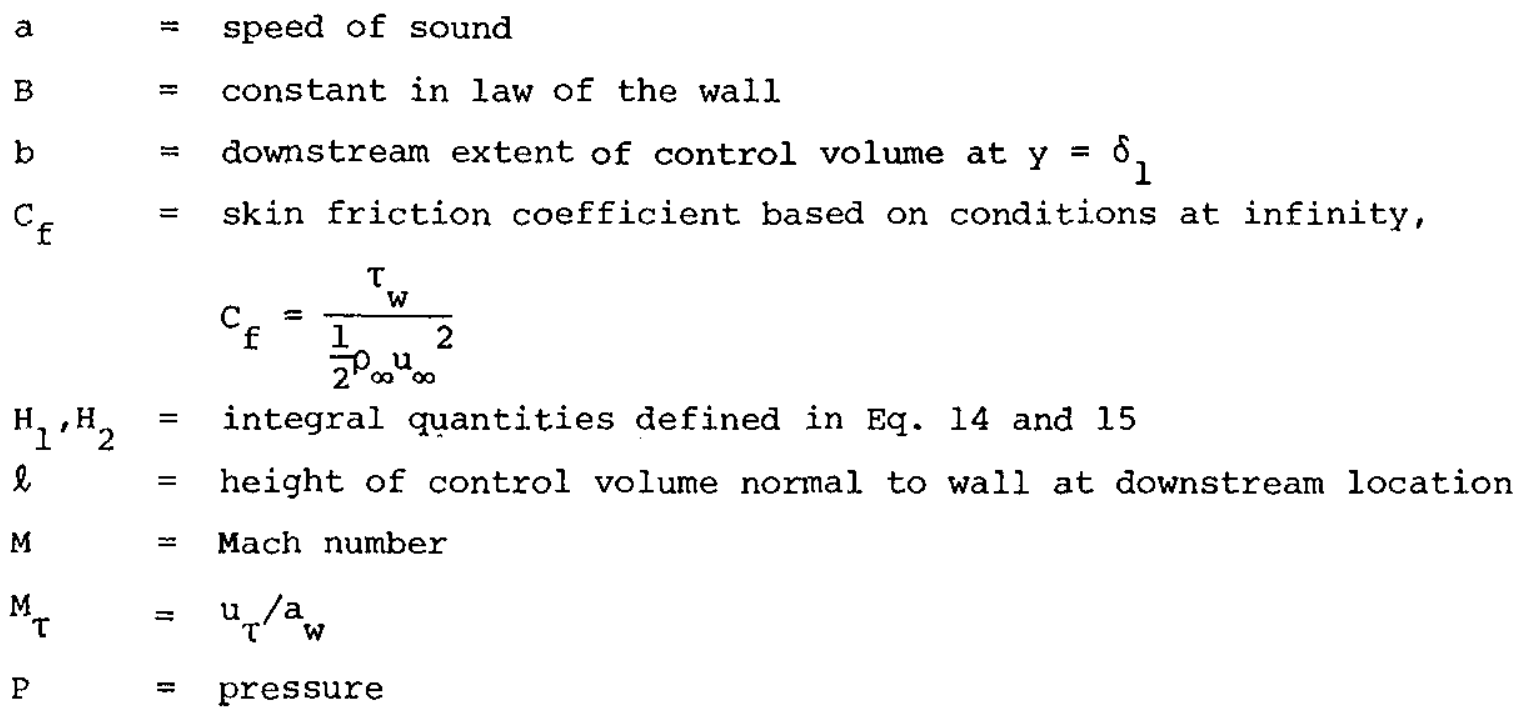

\footnotetext{
*Senior Engineer/Scientist, Member AIAA, Current Address: Rockwell International/Rocketdyne Division, Canoga Park, California

**Consultant, also Professor, California Institute of Technology, Pasadena California, Fellow AIAA

$\dagger_{\text {Engineer/Scientist }}$
} 


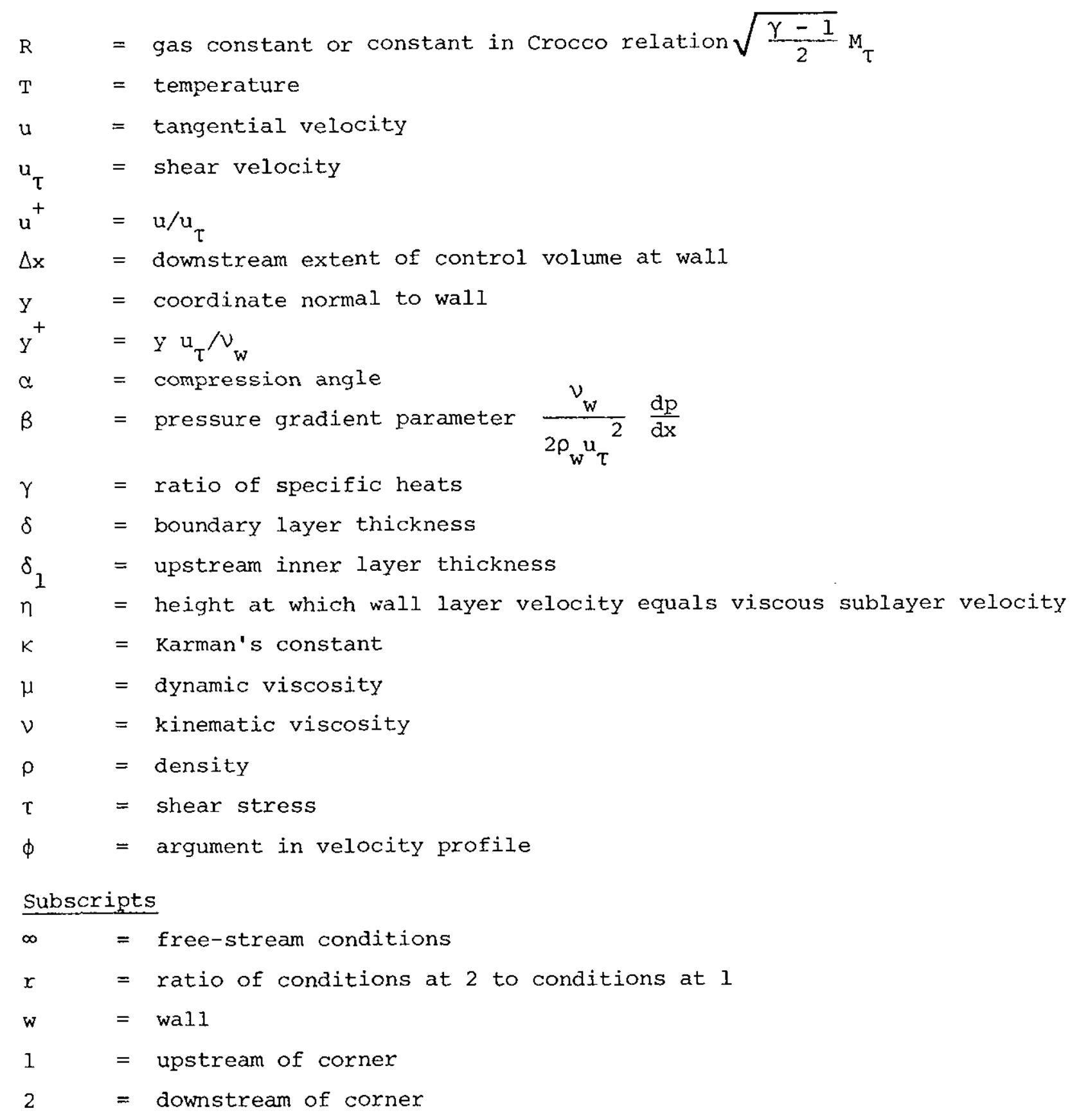




\section{Introduction}

It has been known for some time (Ref. 1, 2, and 3) that an inviscid technique can be used to predict part of the pressure distribution that results when a nonseparating turbulent boundary layer interacts with a two-dimensional compression corner. In this technique, the effect of the inner part of the boundary layer profile is ignored. The outer part is treated as an inviscid rotational supersonic shear flow with a supersonic slip velocity at the wall (i.e., at the outer edge of the inner, thin, neglected portion of the profile). Corresponding to a given slip Mach number $\left(M_{w}\right)$ and corner angle $(\alpha)$, there is an initial pressure jump followed by a pressure distribution on the ramp, which can be accurately determined by a method of characteristics solution (Ref. 1, 2, and 3). The initial step, the choice of $\mathrm{M}_{\mathrm{W}}$, is crucial in obtaining the rest of the solution but there has been no rational basis for making the selection.

Figure 1 shows the typical pressure distribution that is observed experimentally for nonseparating corner flow at sufficiently high Reynolds number and Mach number. At the beginning of the pressure rise, the gradient is so steep that it is not resolved by the limited distribution of pressure measuring points and looks almost like a jump. This is followed by a more gradual rise toward the value determined by the simple global relations for supersonic flow over a ramp. The existence of these two different regions in the pressure distributions implies that there are two regions in the boundary layer in which the pressure $x$ ise is controlled by different mechanisms. It clearly suggests the two-layer model already implied in the inviscid calculations described above and indicates that the mechanism for the initial

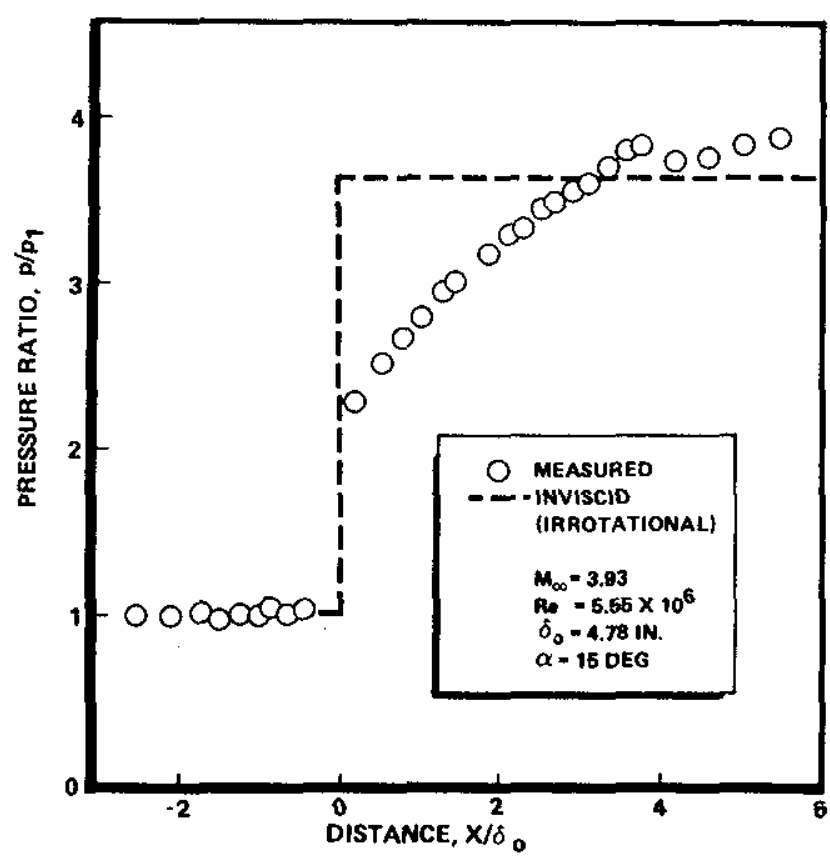

Fig. 1. Surface pressure distribution on an unseparated compression corner

pressure rise must be sought in the inner layer. An important part of the problem is to define the inner layer, i.e., its thickness. In the work described below, we do this by applying a momentum integral analysis to the inner layer and matching the pressure rise in it to that in the outer layer. The mechanism for pressure rise in the outer layer is the oblique shock but in the inner layer it is a more complicated process, possibly involving mixing as well as shock processes. We avoid the problem of addressing the details by using a momentum integral method. 
The division of the boundary layer into two layers, an outer inviscid rotational supersonic layer, as in the previous inviscid methods, and a thin, inner wall layer is shown in Fig. 2. Here, the inner layer is shown in expanded scale for clarity. In the outer layer, the flow is calculated by the method of characteristics; the influence of shear stress is neglected. In contrast to usual boundary layer theory, the pressure is not uniform across the outer layer but propagates along the characteristic Mach lines. It is assumed that the inner layer is governed by boundary layer equations except in the vicinity of the corner, and, in particular, that the pressure across it is uniform. Thus, the pressure at the inner edge of the outer layer is transmitted through the inner layer to the wall. Pressure and velocity continuity are required at the interface of the two layers, and these conditions determine the location of the interface. (In some cases, the velocity matching requirement is relaxed.)

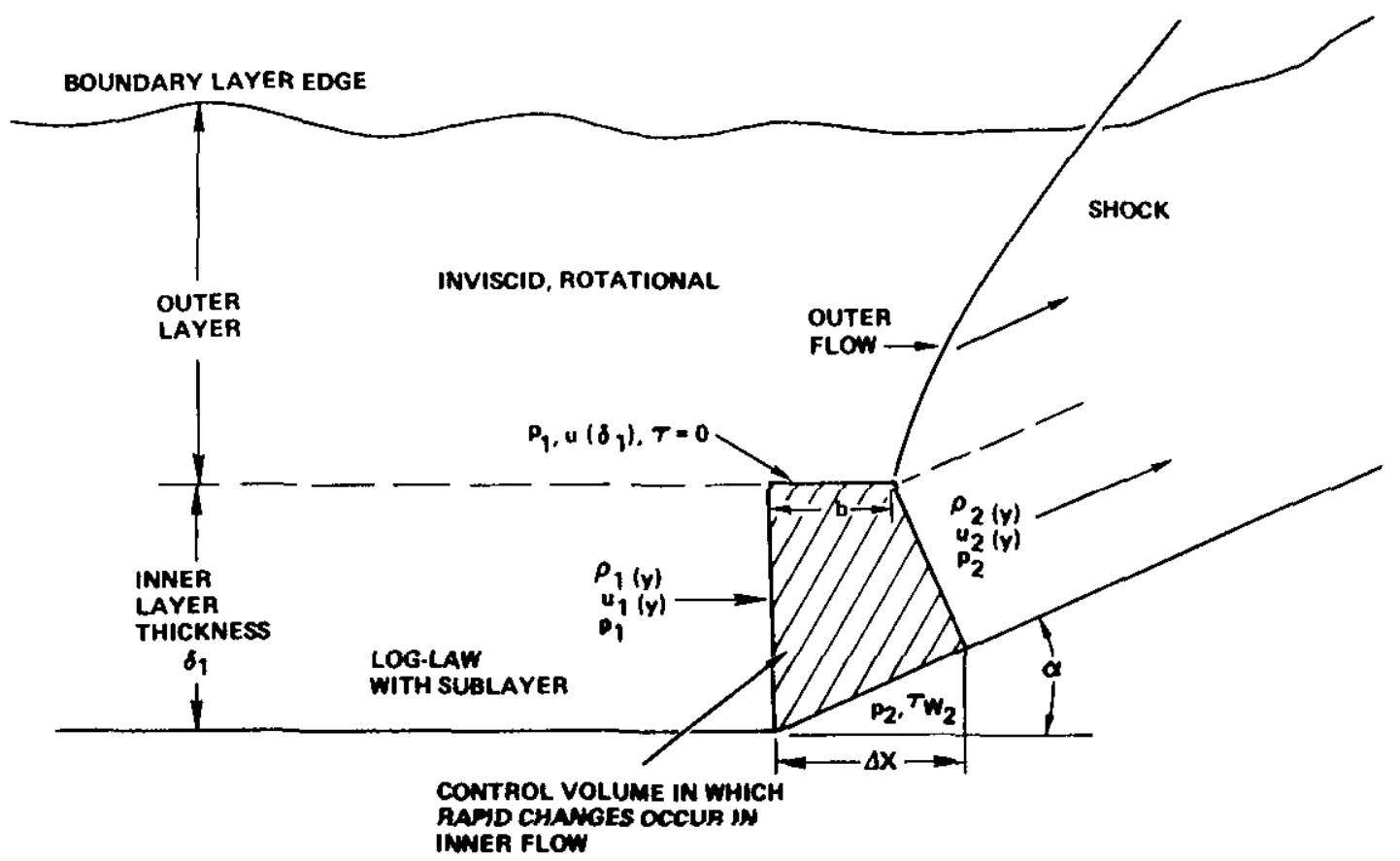

Fig. 2. Sketch of boundary layer showing two layers and control volume

\section{Analysis}

The analysis necessary to obtain solutions from our flow model is presented in this section. The governing equations for the inner layer are obtained and the matching process between the two layers explained. Velocity profiles are necessary inputs in our formulation and they are introduced. The resulting algebraic equations can be readily solved providing the dependence of the solution on the inner layer thickness is known. This dependence is illustrated. 
Before beginning the derivation of the equations, it is informative to look at two features of the control volume shown in Fig. 2. These are its streamwise extent, $\Delta \mathrm{x}$, and the placement of the shock wave. Usually the extent of a control volume is arbitrary and either drops out of the final equations or the desired results are obtained in the limit as $\Delta x$ approaches zero. Neither of these hold true in the present instance. The length, $\Delta x$, of the control volume is part of the solution, i.e., one of the unknowns to be determined. Later, it will be shown that an explicit relation exists between $\mathrm{p}_{r}$ and $\Delta \mathrm{x}$.

The shock is assumed to emanate from the upper right corner of the control volume as shown in Fig. 2. Above this point, the shock is well defined with the usual properties of a simple oblique shock. Below, within the control volume, its form may be more complex and other dissipative processes, e.g., mixing, may be occurring, but need not be described in detail.

We begin the derivation of the equations by considering the forces and fluxes on each face of the control volume.

The upstream influence of the interaction, as indicated by experimental data, is very small for nonseparating flows. Because of this and by the placement of the shock, the upstream and top faces of the control volume are assumed to be in the undisturbed boundary layer flow. Thus, conditions at these surfaces are known. The shear stress acting on the upper surface is taken to be zero since it is adjacent to the outer inviscid layer.

The downstream face is in the region affected by the corner flow so its flow properties are unknown and to be determined. This face is slanted to the free stream but normal to the downstream wall so that the tangential velocity, $u_{2}(y)$, can be expressed, as usual, in terms of the wall normal coordinate. The pressure along this face is also constant since it is within the inner layer, but all the other quantities vary with height.

On the wall surface, conditions are taken to be constant and equal to theix values at the downstream edge. Since conditions actually vary continuously from upstream to downstream, some mean value would be more appropriate. However, we found that the main effect of introducing a mean value in the formulation, e.g., $1 / 2\left(p_{1}+p_{2}\right)$ for the pressure on the wall, is to change the solution in $\Delta x$ and not in the pressure rise $p_{2}-p_{1}$. Therefore, we retained the simpler form.

The conservation of mass in the control volume gives:

$$
\int_{0}^{\delta} \rho_{1} u_{1} d y=\int_{0}^{l} \rho_{2} u_{2} d y
$$

In both of these integrals, the integration is carried out normal to the respective surface. The upper limit, $\ell$, of the second integral is related to $\delta_{1}, \alpha$, and $\Delta x$ by simple geometry 
The conservation of momentum in the direction of the initial flow gives:

$$
\left(p_{1}-p_{2}\right) \delta_{1}-\tau_{w_{2}} \Delta x=\int_{0}^{\ell} \rho_{2} u_{2}^{2} \cos \alpha d y-\int_{0}^{\delta_{1}} \rho_{1} u_{1}{ }^{2} d y
$$

In the direction normal to the incoming flow, the momentum equation is:

$$
\left(p_{2}-p_{1}\right) b-\tau_{w_{2}} \Delta x \tan \alpha=\int_{0}^{l} \rho_{2} u_{1}^{2} \sin \alpha d y
$$

where $b$, like $\ell$, can be determined from the geometry.

In place of an energy equation, we assume a crocco relation:

$$
\frac{\rho}{\rho}=1-R^{2}\left(\frac{u}{u_{\tau}}\right)^{2}
$$

where

$$
R^{2} \equiv \frac{\gamma-1}{2} M_{\tau}^{2}=\frac{Y-1}{2}\left(\frac{u_{v}}{a_{w}}\right)^{2}
$$

Equation 4 implies an adiabatic wall and turbulent PrandtI number of unity.

The equation of state for a perfect gas,

$$
\mathrm{p}=\rho \mathrm{RT}
$$

coupled with the fact that the wall temperature is constant, implies $\left(\rho_{\mathrm{w}}\right)_{\mathrm{r}}=$ ( $p)_{x}$ where the notation indicates the ratios $\rho_{w_{2}} / \rho_{w_{1}}$ and $p_{2} / p_{I}$.

A simple relation for the pressure ratio can be obtained by eliminating the downstream momentum flux from Eq. 2 and 3.

$$
\frac{p_{2}}{p_{1}}=1+\frac{\sin \alpha \cos \alpha}{\Delta x} \int_{0}^{\delta} \rho_{1} u_{1}^{2} d y
$$

This shows that not only does the streamwise extent of the control volume remain in the problem but that the pressure rise is coupled to it. It is one of the variables that is determined in the solution simultaneously with the others. 
This integral formulation of the inner layer requires that a functional relation be assumed for the velocity distributions. The upstream distribution is known but the proper assumption for the downstream profile shape is not so clear. Two different downstream families were tried. A family of linear profiles with slip at the wall gave solutions that did not agree well with the experimental results, presumably because this shape is too restrictive. A family of downstream profiles, which is related to the upstream profiles, gives much better results and is described in what follows.

The upstream, undisturbed profile in the wall layer is assumed to be given by the Van Driest transformed law of the wall, joined to a linear velocity distribution in the viscous sublayer. For the downstream profiles, the same family is used but a pressure gradient parameter is included to allow for more latitude in the shape. This is introduced as follows. The velocity in the viscous sublayer can be expressed as:

$$
\mathrm{u}^{+}=\mathrm{y}^{+}+B \mathrm{y}^{+2}
$$

where

$$
\beta=\frac{v_{w}}{2 \rho_{w} u_{\tau}^{3}} \frac{d p}{d x}
$$

Using mixture length formulas and the Crocco relation, Eq. 4, it is possible to obtain the Van Driest form of the law of the wall (see, for example, Ref. 4). If the pressure gradient is retained in the momentum equation, the following expression for the velocity in the wall layer results.

$$
\mathrm{u}^{+}=\frac{1}{\mathrm{R}} \sin \left\{\frac{2 \mathrm{R}}{\mathrm{K}}\left[\sqrt{1+2 B y^{+}}-I\right]+\frac{\mathrm{R}}{\mathrm{K}} \ln \left[\frac{2}{\beta} \frac{\sqrt{1+2 \beta \mathrm{y}^{+}}-1}{\sqrt{\mathrm{I}+2 B \mathrm{y}^{+}}+1}\right]+\mathrm{RB}\right\}
$$

In obtaining Eq. 8, the constant of integration was chosen so the usual result

$$
u^{+}=\frac{1}{R} \sin \left\{\frac{R}{K} \ln y^{+}+R B\right\}
$$

is obtained in the limit as $\beta \rightarrow 0$. It should be emphasized that $\beta$ is being used here as a profile shape parameter. Its relation to the actual pressure gradient in the nonequilibrium corner region is uncertain. Similarly, $u_{\tau}$ appears as another parameter whose relation to the actual shear stress downstream of the corner is also uncertain.

Upstream of the corner, Eq. 7 with $\beta=0$ is assumed to hold inside the viscous sublayer and $\mathrm{Eq}$. 9 to hold outside. The value at which the velocity changes from Eq. 7 to Eq. 9 is obtained by simultaneous solution of Eq. 7 and 9. In a like manner downstream of the cornex, the point at which the governing relation changes from Eq. 7 to 8 is obtained by their simultaneous solution. 
Substitution of the velocity profiles into Eq. 1, 2, and 6, respectively, results in the following three algebraic equations for the three unknowns, $p_{r}$ ' $u_{\tau_{r}}$, and $\Delta x$ in terms of $\delta_{1}$ and the parameter $\beta$.

$$
\begin{aligned}
& -\frac{1}{2} \ln \left(1-R_{1}^{2} \eta_{1}^{+2}\right)+R_{1} \int_{\eta_{1}^{+}}^{\delta_{1}^{+}} \tan \phi_{1} \sec \phi_{1} d y^{+} \\
& -\mathrm{R}_{1}^{2} \int_{0}^{\mathrm{n}_{2}^{+}} \frac{\left(y^{+}+\beta_{y}^{+{ }^{2}}\right)^{2} d y^{+}}{1-\mathrm{R}_{1}{ }^{2} \mathrm{u}_{\tau_{x}} 2\left(y^{+}+\beta y^{+}\right)^{2}} \\
& -\frac{\mathrm{R}_{1}}{\mathrm{u}_{\tau_{r}}} \int_{\eta_{2}^{+}}^{\ell^{+}} \tan \phi_{2} \sec \phi_{2} d y^{+}=0 \\
& \frac{\gamma-1}{\gamma}\left(1-p_{r}\right) \delta_{1}^{+}-u_{\tau_{r}}{ }^{2} p_{r} R_{I}^{2} \Delta x_{1}^{+}+H_{1}-\frac{H_{2}}{u_{\tau_{r}}} \cos \alpha=0 \\
& \mathrm{p}_{r}=1+\frac{2 \gamma}{\gamma-1} \frac{\sin \alpha \cos \alpha}{\Delta \mathrm{x}_{1}^{+}} \mathrm{H}_{1}
\end{aligned}
$$

where $\phi_{1}$, is the argument of the sine function in Eq. $9, \phi_{2}$ is the argument in Eq. $8, l^{+}$is found fxom the geometry of Fig. 2 as:

$$
\ell^{+}=u_{\tau_{r}} p_{r} \frac{\delta_{1}^{+}-\Delta x_{1}^{+} \tan \alpha}{\cos \alpha}
$$

and the H's are defined as:

$$
H_{1}=-\eta_{1}^{+}+\frac{1}{2 R_{1}} \ln \frac{\left(1+R_{1} \eta_{1}^{+}\right)}{\left(1-R_{1} \eta_{1}^{+}\right)}+\int_{\eta_{1}^{+}}^{\delta_{1}^{+}} \tan ^{2} \phi_{1} d y^{+}
$$




$$
\mathrm{H}_{2}=u_{\tau_{r}}^{2} \mathrm{R}_{1}^{2} \int_{0}^{\eta_{2}^{+}} \frac{\left(\mathrm{y}^{+}+\beta y^{+2}\right)^{2} d y^{+}}{1-u_{\tau_{x}}{ }^{2} \mathrm{R}_{1}^{2}\left(y^{+}+\beta y^{+}\right)^{2}}+\int_{\eta_{2}^{+}}^{e^{+}} \tan ^{2} \phi_{2} d y^{+}
$$

There are now three equations for five unknowns: $p_{x}, u_{\tau_{r}}, \Delta x, \delta_{1}$, and $\beta$. The required two additional relations come from matching pressure and velocity at the interface between the two layers.

The innex layer flow leaving the control volume has been turned through the compression angle $\alpha$. The same turning angle is assumed for the streamline at the inner edge of the outer layer. Knowing the turning angle, it is a simple matter to calculate the flow properties across the shock. This determines conditions in the outer layer at the point where the shock leaves the control volume. Compatibility between the layers requires that the innex and outer pressures and velocities at this point be equal. Closure of the problem is obtained by imposing this compatibility condition and a unique solution results.

Once the solution is obtained, $p_{r}$ and $u_{\tau_{r}}$ are known. The change in skin friction can then be found:

$$
c_{f_{r}}=p_{r} u_{\tau_{r}} 2
$$

where $C_{f}$ is defined in terms of the free-stream quantities, However, since $\tau_{w}$ was introduced mainly as a profile shape parameter, and could have been omitted from the momentum equation, as mentioned earlier the significance of the resulting value of $\mathrm{C}_{f}$ is uncertain.

A typical solution curve for Eq. 10 through 12 is shown in Fig. 3 as the solid line. The pressure rise, $p_{r}$, is plotted as a function of $M\left(\delta_{1}^{+}\right)$, which is equivalent to plotting $p_{r}$ against $\delta_{1}^{+}$. The shape of the curve is reminiscent of the well known result for pressure rise across an oblique shock (Ref. 5). This is not surprising since these two cases are closely related. The present result differs from the usual calculation only in that we replaced the uniform velocity profile with a nonuniform one, while retaining a uniform pressure profile. Also shown in this figure, as the dashed curve, is the irrotational oblique shock pressure rise obtained from conditions at the innex edge of the outer layer. The overall solution for a particular set of freestream conditions occurs at the intersection of the two curves (providing of course that the velocities at the edges of the two layers axe also equal, as they are for the case shown with $B=5.0$ ). It is interesting that the intersection always occurs along the lower branch of the curve, just as the weak branch of the oblique shock solution is the one always observed. 


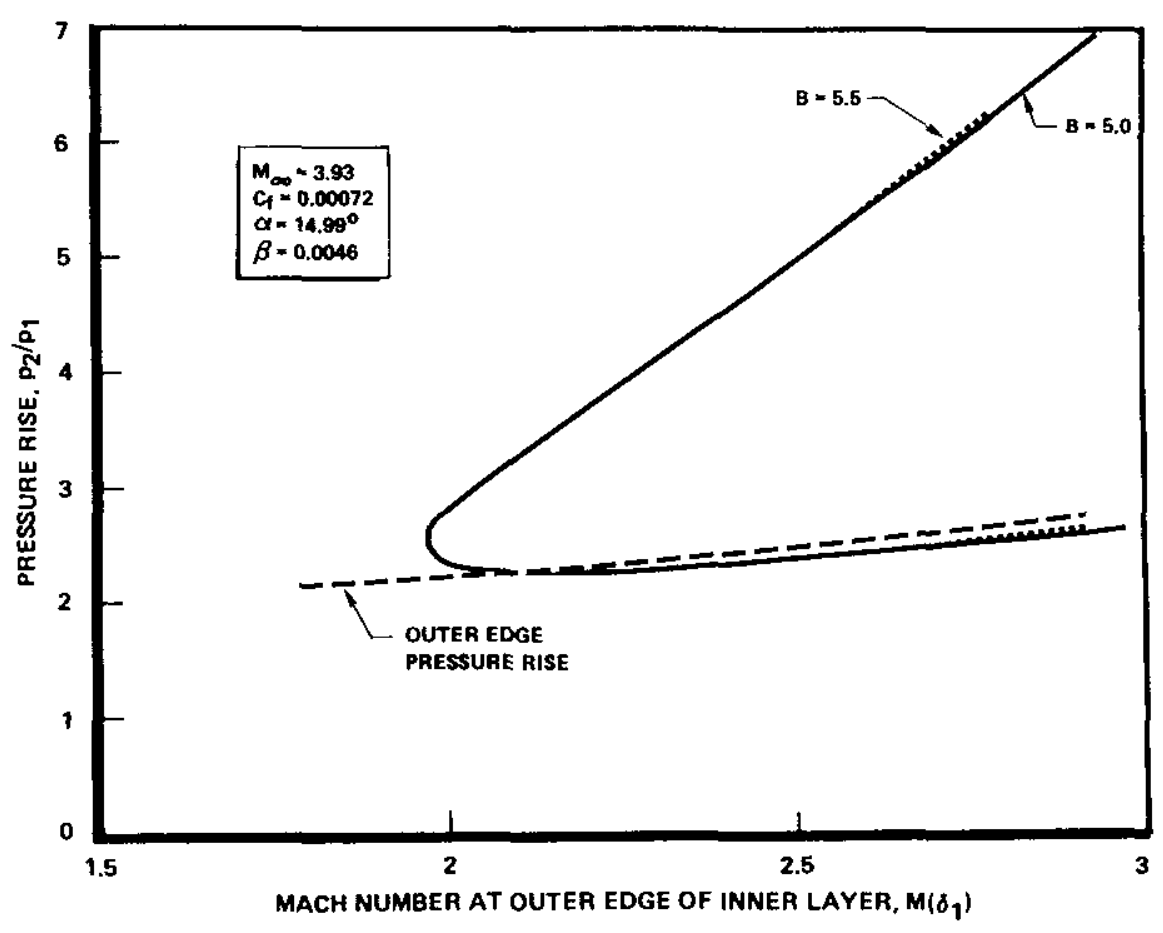

Fig. 3. Typical solution

Because of the simplicity of Eq. 10 through 12 , solutions are readily obtained. For example, it took about 10 seconds on the CYBER 174 to generate all the data for Fig. 3. A particular solution can be obtained in less than onehalf that time.

\section{Results}

In this section, we compare the results with experimental data to illustrate the accuracy of the method. In developing the method and becoming familiar with its limitations, calculations were made for a wide range of conditions. Data do not exist for many of the conditions but where they do, comparisons were made. The three references that were used primarily are the papers by Roshko and Thomke (Ref. 2), Elfstrom (Ref. 3), and Settles, Fitzpatrick, and Bogdonoff (Ref. 6).

The results of the comparisons are summarized in Table 1 . The freestream Mach number, skin friction coefficient, and compression angle are given for each case along with the reference numbex of each source of data. The calculated pressure rise, which occurs $\Delta \mathrm{x}$ downstream of the cornex, is given and comparison is made to the measured pressure at this same downstream point. Finally, the value of $\delta_{l}$ and the value of $\Delta x$ are presented in terms of $\delta$. The cases with the asterisk correspond to calculations made with velocity slip with $\beta=0$ and will be discussed later. It can be seen that good agreement exists for most cases and in some instances the agreement is exceptional. 
Table 1. Comparison of Measured and Predicted Corner Pxessure Jump

\begin{tabular}{|c|l|l|l|l|l|l|l|}
\hline $\begin{array}{l}\text { DATA } \\
\text { SOURCE, } \\
\text { REF. }\end{array}$ & $M_{\infty}$ & \multicolumn{1}{|c|}{$\mathrm{C}_{\mathrm{f}}$} & $\alpha$ & $\begin{array}{l}\text { MEASURED } \\
\text { PRESSURE }\end{array}$ & $\begin{array}{l}\text { CALCULATED } \\
\text { PRESSURE }\end{array}$ & $\frac{\Delta \mathrm{x}}{\delta}$ & $\frac{\delta_{1}}{\delta}$ \\
\hline 2 & 2.995 & 0.00094 & 14.88 & 2.24 & 2.21 & 0.2311 & 0.2425 \\
2 & 3.93 & 0.00068 & 18.55 & 2.69 & 2.80 & 0.1139 & 0.123 \\
2 & 3.93 & 0.00072 & 14.99 & 2.23 & 2.27 & 0.0918 & 0.0951 \\
2 & 3.93 & 0.00072 & 14.99 & 2.23 & $2.20^{*}$ & 0.0542 & 0.0617 \\
2 & 3.93 & 0.00072 & 20.03 & 2.90 & 3.10 & 0.158 & 0.1735 \\
2 & 4.92 & 0.0006 & 14.86 & 2.22 & 2.33 & 0.0785 & 0.0782 \\
2 & 4.92 & 0.0006 & 19.97 & 2.90 & 3.22 & 0.1162 & 0.1251 \\
3 & 9.22 & 0.0002157 & 15.00 & 2.90 & 2.40 & 0.0235 & 0.0235 \\
3 & 9.22 & 0.0002157 & 26.0 & 3.90 & $4.64^{*}$ & 0.0408 & 0.0408 \\
6 & 3.0 & 0.001 & 8.0 & 1.47 & 1.52 & 0.1535 & 0.1491 \\
6 & 3.0 & 0.001 & 16.00 & 2.15 & 2.37 & 0.303 & 0.320 \\
\hline
\end{tabular}

*DENOTES VELOCITY SLIP

The worst agreement was with Elfstrom's experiment because it was difficult to obtain an estimate of the initial pressure rise from his data. This estimate has to be made by extrapolating the downstream pressure distribution back toward the cornex. Some scattex in the data and a relatively sparse distribution of experimental points (Fig. 4) made this extrapolation difficult. Consequently, the experimental values given in Table 1 , which was made before the calculated result was available, are apparently in error. This may be inferred from Fig. 4 where the calculated pressure distribution gives a good fit to the experimental data for $\alpha=15$ degrees. For $\alpha=26$ degrees, the discrepancy in Table I may also come from the theoretical value which was calculated with $\beta=0$.

All of the results in Table 1 are for high values of $R_{x}$. It is expected that better results occur at these Reynolds numbers because the inner layer thickness decreases as Re increases.

To get a better appreciation of the accuracy of the results presented in Table 1 , a more meaningful

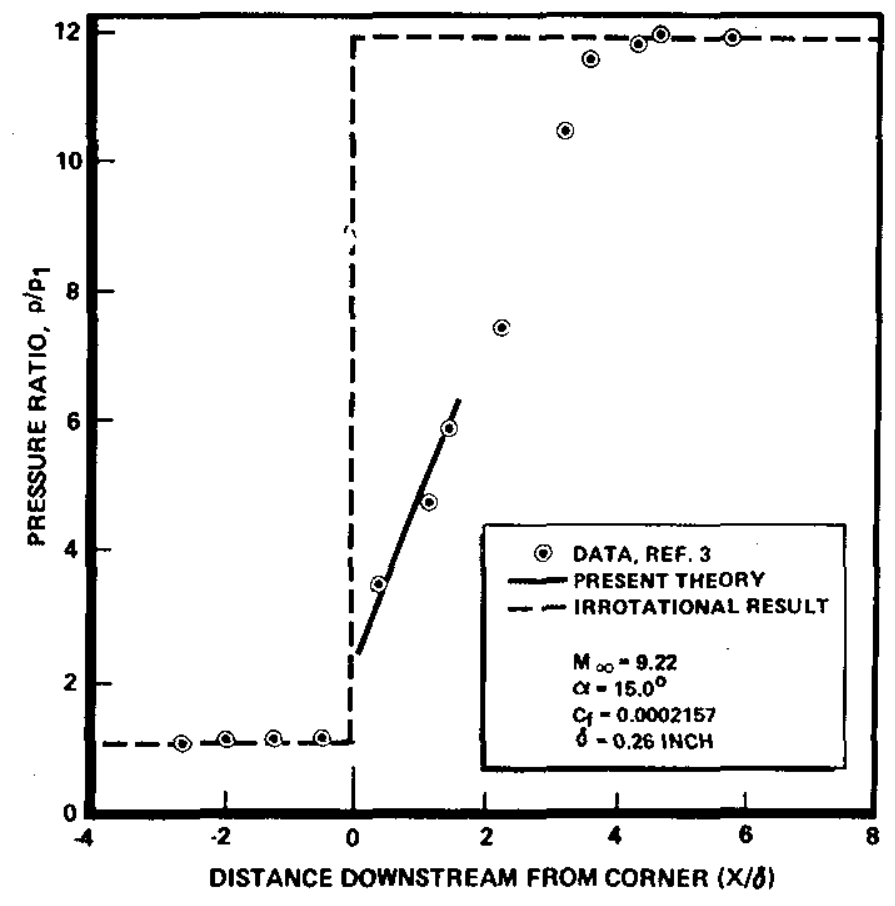

Fig. 4. Comparison of experimental and calculated pressure distributions 
representation is given in Fig. 5 and 6 where the calculated initial pressure rise is compared with experimental pressure distributions. The two cases shown are the first two of Table 1. They are at two different Mach numbers and compression angles. The agreement is seen to be quite good. Three other similar comparisons for other conditions are shown in Fig. 4, 7, and 8, where the initial pressure rise now appears as the first point in a calculated pressure distribution. These include one of Elfstrom's cases discussed above, and the agreement appears as good for Fig. 4, 7, and 8 as it does for Fig. 5 and 6 .

Based upon the results presented in Table 1 and Fig. 4 through 8 , we conclude that the method presented here provides an accurate prediction of the initial point of the pressure distribution.

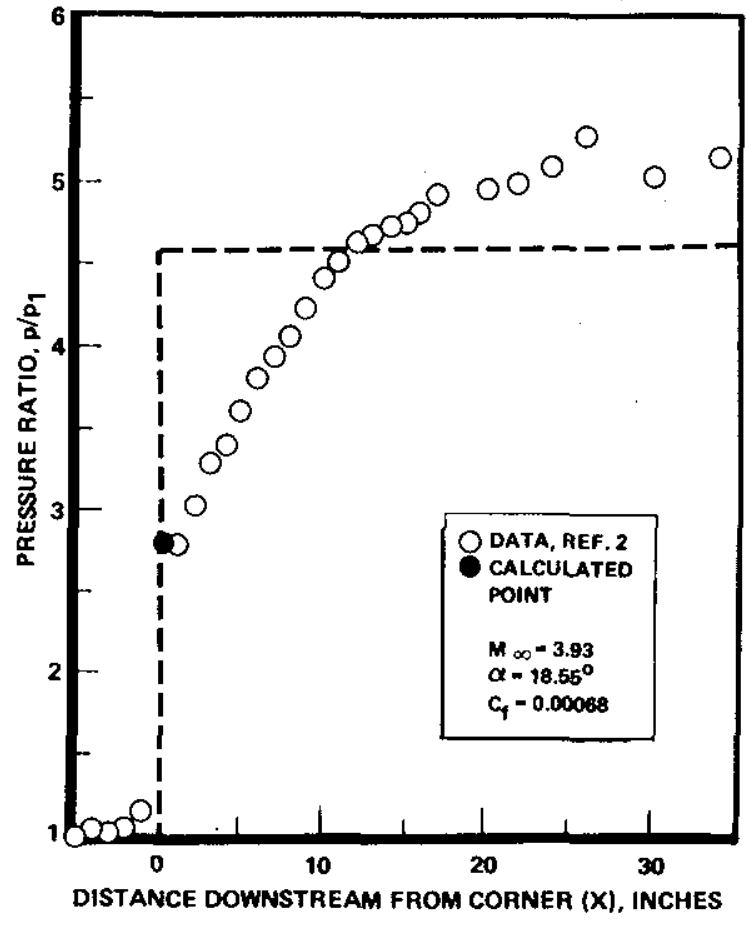

Fig. 5. Pressure distribution of Ref. 2 with calculated point included

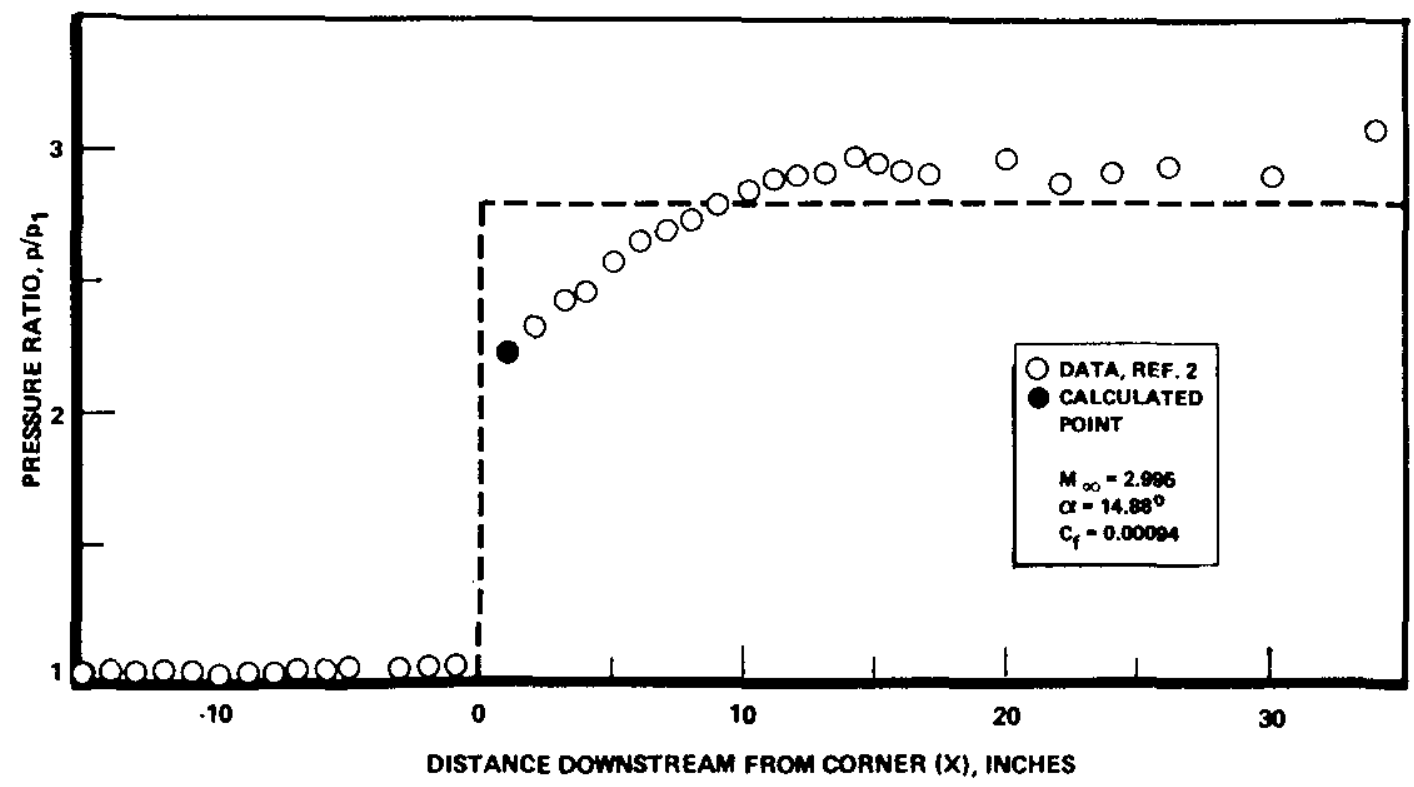

Fig. 6. Pressure distribution of Ref. 2 with calculated point included 


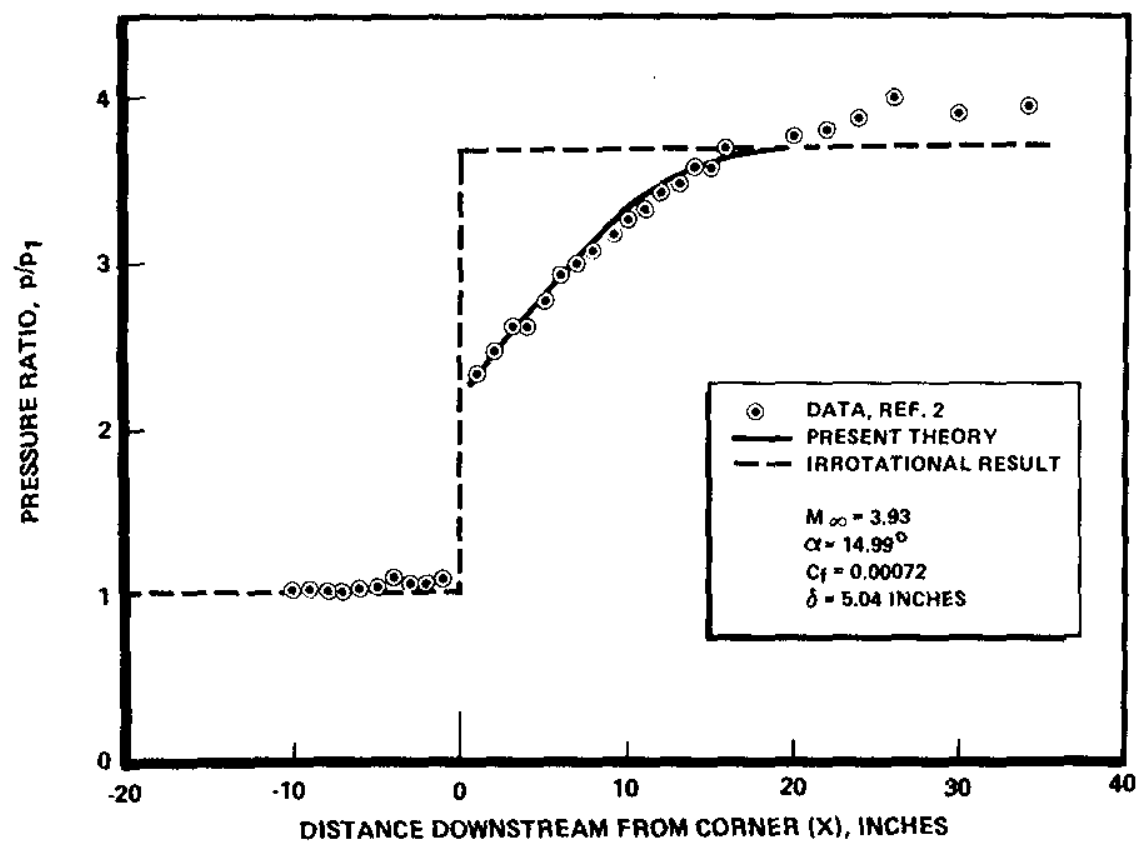

Fig. 7. Comparison of experimental and calculated pressure distributions

It is interesting to observe the effect of Reynolds number and edge Mach number on the pressure rise. These two effects are shown in Fig. 9 and 10. Figure 9 is a plot of pressure rise as a function of compression angle for different values of the skin friction coefficient. The curves cover a Reynolds number range from $2 \times 10^{6}$ to $5 \times 10^{9}$. The curve for $\mathrm{C}_{\mathrm{f}}=$ 0.0015 is presented for completeness, but at such low Reynolds numbers the calculation violates the thin inner layer assumption and may be in error. Everywhere along this curve $\delta_{1} / \delta>0.62$. At the two higher Reynolds numbers, where the results are more valid, the effect of Reynolds number is quite smal1. The effect of compression angle on the other hand, is large. Doubling $\alpha$ from 10 to 20 degrees more than doubles the pressure jump. The same type of

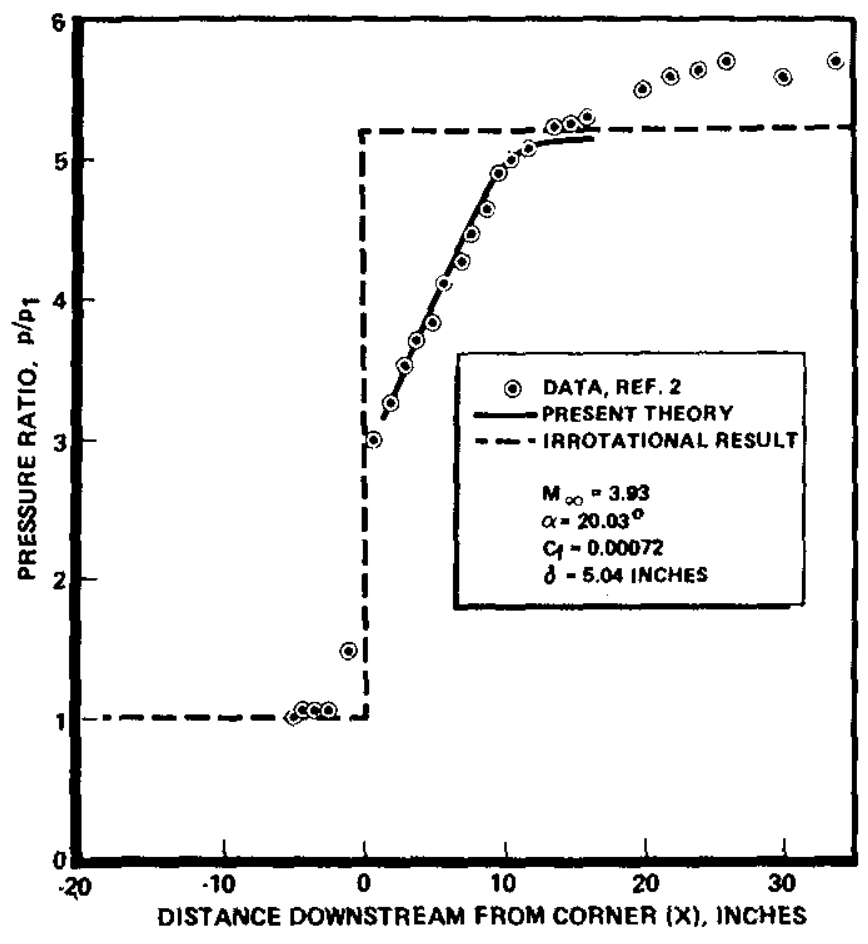

Fig. 8. Comparison of experimental and calculated pressure distributions 


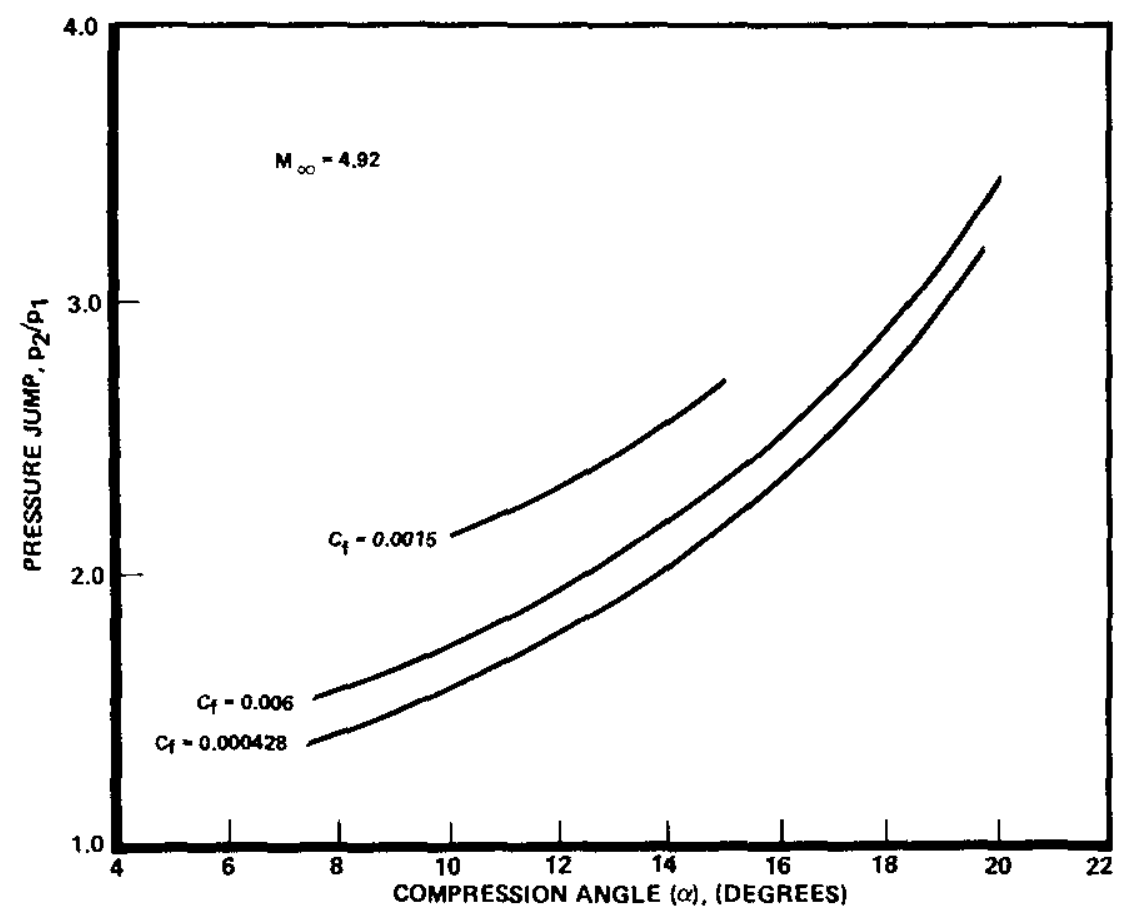

Fig. 9. Effect of compression angle and skin friction on pressure jump result is found in Fig. 10, where the dependence on $\mathrm{M}_{\infty}$ is shown at constant $\mathrm{Re}_{\mathrm{X}}$. Pressure $\mathrm{rise}$ is again plotted as a function of compression angle for three values of $M_{\infty}$, namely 3,5 , and 9. The pressure rise variation with Mach number is surprisingly small, its largest difference being 10\% for Mach number changes of more than a factor of 3 .

The eventual purpose of this work is the calculation of the entire downstream pressure distribution, not just a single point. A step in this direction can be taken by using the inviscid method (Ref. 1, 2 and 3 ) described in the Introduction, but now using for the initial point the value calculated by the present method rather than the ad hoc values previously used.

The results of three such calculations are presented in Fig. 4, 7 , and 8. The last two are for conditions that correspond to data obtained by Roshko and Thomke (Ref. 2),

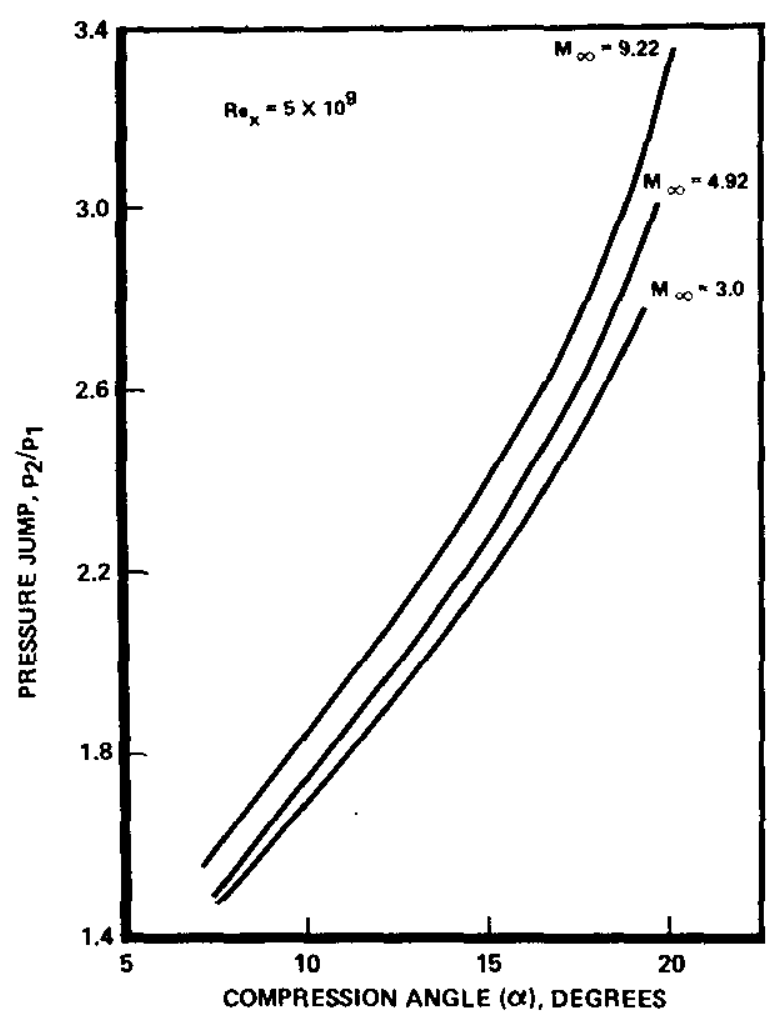

Fig. 10. Effect of compression angle and mach number on pressure jump 
$M_{\infty}=4, C_{f}=0.0007$, and the first for conditions observed by Elfstrom (Ref. 3), $M_{\infty}=9, C_{f}=0.0002$. The first two cases axe for compression angles of 15 degrees and the third is for 20 degrees. For the first case, for $M_{\infty}=9$, the calculation stopped when the pressure ratio at the wall reached a value of 6.1 because the characteristics method could not be continued to the next grid point, which would be at a subsonic condition. ( $\mathbb{M}_{\infty}=1$ corresponds to $\mathrm{p}_{2} / \mathrm{p}_{1}=7.2$ in this example.) In the last two cases, the calculation was able to proceed quite far downstream and it is obvious that it will be asymptotic to the oblique shock value.

The data are shown by the circles, the calculation by the solid curve, and the dashed line is what one gets from an irrotational oblique shock calculation. In each case, the agreement is good until either the data rise above the oblique shock value or the calculation stops.

\section{Discussion}

The two-layer model we have introduced has premises similar to earlier analyses of boundary layers subjected to sudden change (Ref. 7 through 10), i.e., viscous (or Reynolds) stresses play no direct role in the development except in some limited region near the wall. Our goal is more limited than these analyses in that we do not seek a complete description of the flow, but only of the pressure rise. Hence, we axe able to use the simple, integral method and avoid consideration of the stresses entirely. If one were interested in furthex details of the flow, such as an accurate skin friction prediction or a determination of the downstream velocity profile, the corner region will have to be solved in much more detail. We do not try to draw any comparisons or analogies with rigorous, asymptotic, multideck models (Ref. 7 through 10). Our treatment, which is akin to that of stratford (Ref. 11) is probably more applicable to flows at finite Reynolds number, Mach number, and pressure jump than an asymptotic analysis would be.

An interesting aspect about the corner flow that comes out of this work has to do with length scales. In all of the cases we ran, the solution gave $\Delta \mathrm{x} \sim \delta_{1}$. This means that the $\mathrm{x}$ and $\mathrm{y}$ length scales are equal and that (almost) the full time-averaged Navier-stokes equations must be used to describe the flow. This equality of length scales in the neighborhood of the corner makes questionable the validity of the thin layer approximation used in many numerical solutions of the time-averaged Navier-stokes equations (see, for example, Ref. 12). In high Mach number, high Reynolds number cases when both $\delta_{1}$ and $\Delta x$ may be of the order of the grid spacing or less, this question is academic. But at more moderate values of $R e$ and $M_{\infty}$ where $\Delta x$ approaches $0.1 \delta$ or $0.2 \delta$ the thin layer approximation should be re-examined.

Now let us consider some particular aspects of the model. For a given $B$, the solution of $\mathrm{Eq} .10$ through 12 determines the parameter $\mathrm{C}_{\mathrm{f}_{2}}$ (or, as it actually appears, $\mathrm{u}_{\tau_{2}}$ ) with $\mathrm{B}$ fixed. There is an alternate formulation in which $B$ is allowed to vary while $C_{f_{2}}$ is kept fixed. This was tried and met with only limited success. Having $\mathfrak{u}_{\tau_{2}}$ as an unknown with $B$ constant defines 
$\mathrm{C}_{\mathrm{f}_{2}}$ and avoids an overspecification inherent in the alternate approach. Whether the quantity $B$ should remain constant or vary is arbitrary since thexe is not enough experimental data for these nonequilibrium flows to offer substantial guidance.

Perhaps the most significant single assumption with respect to obtaining results is that of the choice of velocity profiles. There should be no controversy about the incoming profile. The boundary layer pxofile based on the Van Driest transformation of the law of the wall, while approximate, is certainly good enough. The downstream profile is another matter. There is very limited data upon which to base a choice. The velocity starts out with a known upstream shape, is deformed in some unknown manner, and eventually returns to its original shape. A reasonable way to model this is to allow the profile to retain as much of its original functional form as possible. Equations 7 and 8 permit this while still allowing the velocity to be affected by two paxameters. If at a later time it becomes clear that a highly nonequilibrium velocity profile, such as exists at the downstream face of the control volume, can better be expressed in another form, the new expression can be incoxporated in the analysis. It would be very surprising if our results were to change significantly. There are some data which substantiate the use of the law-of-the-wall profiles. Settles (Ref. 13) has some preliminary data taken just downstream of an 8-degree corner that shows a logarithmic behavior for a portion of the profile near the wall. As limited as this information is, it at least provides some justification for the use of $\mathrm{Eq} .7$ and 8 .

It is interesting that the linear profiles did not give good results. The linear profiles were chosen because they offer the simplest two parameter family, not because we thought they represent the actual flow in any special way. It was hoped that resonable solutions could be obtained with these simple profiles. If they could, it would mean that the accuracy of the solution does not depend strongly on the profile. Apparently, this was too much to expect. What this shows instead is that the profile family must be chosen with some care. No attempt was made to determine if a profile more general than the linear ones, such as quadratic profiles, would give reasonable answers. To do this would perhaps prove very interesting.

Some additional work was done to determine the importance of the profiles on the results. Figure 3 shows the effect of B to be extremely small, which is comforting. By varying $B$ the momentum is redistributed in the incoming flow although over the range that $B$ was varied this redistribution is not large. In this case, one hopes the results are not strongly affected and it is good to know that they are not.

In still another investigation, the possibility of relaxing the velocity matching condition was explored. In that case, one of the profile parameters $(e . g ., \beta)$ can be dropped (set equal to zero). The effect of doing this is 
illustrated in Fig. 11, which shows the result of choosing various values of $\beta$ between $\beta=0$ and $\beta=0.0046$. At the latter value velocity matching is achieved, while for $\beta=0$, the slip velocity is about $15 \%$. The difference in the corresponding solutions for the pressure rise is only $3 \%$ as may be seen from Fig. 11 or Table 1 .

There may be a case for relaxing the velocity matching condition and extending the range of attainable solutions. Thus, for Elfstrom's (Ref. 3) 26degree case, one which does not have a solution with matched velocities, a solution was readily found. This result perhaps indicates that a slip layer does in fact exist. In a sense, allowance for slip

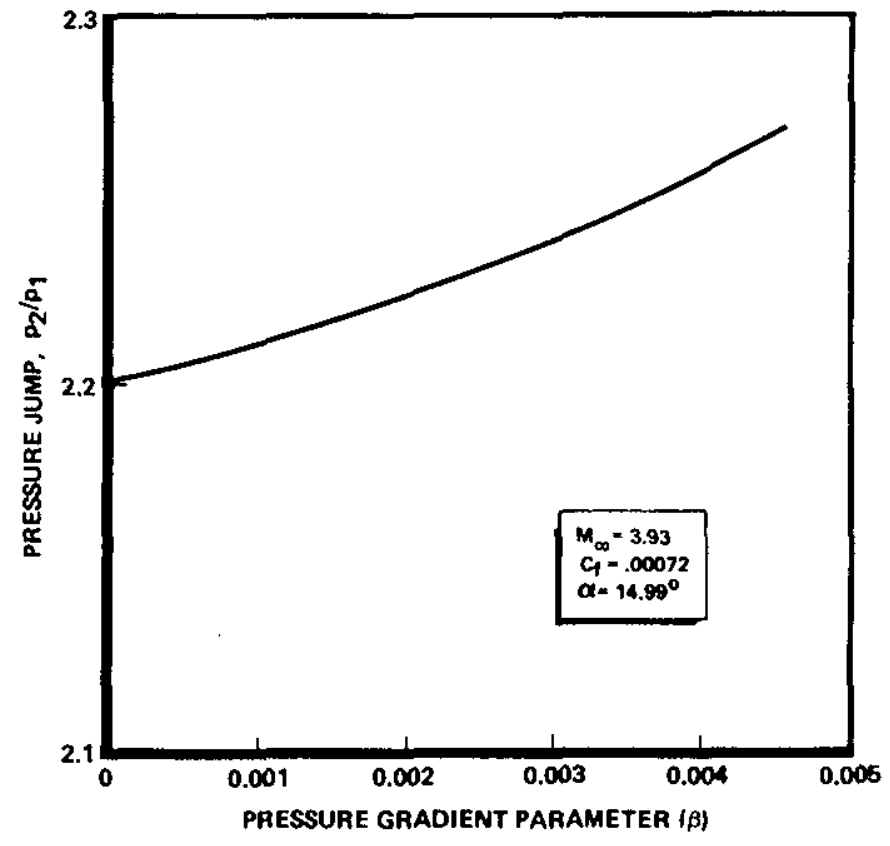

Fig. 11. Effect of $B$ on pressure rise extends the range of profile shapes to include a discontinuity (which in a more complete analysis could be treated as a region that could be smoothed out by introducing and intermediate, third layer).

It is possible to obtain solutions for most meaningful cases. However, there are three limits for which the method breaks down and solutions cannot be found, namely low Reynolds number, high corner angle, or low Mach number. At low values of the Reynolds number, the thickness, $\delta_{1}$, becomes excessive violating the assumptions that it is small and the velocity is propexly described by Eq. 7 and 8. For example, for $M_{\infty}=4.92, \alpha=10$ degrees, and $C_{f_{1}}=0.0015$, corresponding to a Reynolds number, $R e_{X}$, of $2 \times 10^{6}$, the solution is found at $\delta_{1} / \delta=0.87$.

A second limit is encountered at high compression angles. In some instances, value of $\beta$ cannot be found which yield velocity matching. In other cases, solutions are found but again are of doubtful validity because the inner layer thickness is large. Neither occurrence seems to correlate with incipient separation angle. For example, a solution could not be obtained at Elfstrom's (Ref. 3) conditions for $\alpha=22.2$ degrees although he reported attached flow at 30 degrees while a solution was found at 25 degrees for conditions at which Roshko and Thomke (Ref. 2) reported separation at 23 degrees.

These two boundaries of the solution domain correspond to physically realistic limits. The boundary that arises due to low Reynolds number can undoubtedly be moved by the inclusion of a wake component in the velocity 
profile. (of course, thexe must be a lower Reynolds number limit connected with transition.) Some upper limit on compression angle is also expected but it was not expected to occur before the incipient separation angle. Although the formulation is not designed to include flow separation, it is not excluded either, so long as its extent is small and remains within the control volume. The reason why a solution is found beyond the incipient separation angle in some cases and not in other is not known.

There is a third boundary at low $\mathrm{M}_{\infty}$. This comes from the dual requirements on the inner layer that it be thin and still supersonic at its outex edge. Clearly, for sufficiently low fxee stream Mach numbers, it is not possible to satisfy both of these conditions. We did not try to find the location of this boundary but it is believed to be between $M_{\infty}=2.0$ and 2.5 .

As noted earlier and as may be seen from Table 1 and Fig. 9 and 10, the initial pressure rise is rather insensitive to Reynolds number and freestream Mach number. This is because changes in these parametexs produce relatively small changes in the shape of the velocity and density profiles in the inner layer. The thickness of that layer is of course stronly affected and the scale of the interaction region connected with the initial pressure rise changes (decreases with increasing Reynolds number or Mach number) but the pressure rise is independent of that thickness except to the extent that the profile shape is affected.

It was also noted by Elfstrom that heat transfer at the wall has little effect on the pressure distribution and his data in Fig. 4 for a cooled wall compares well with our calculation for an adiabatic wall. This seems at first surprising because, with wall cooling, there is a strong effect on the shape of the profiles, especially the density profile, in the inner layer. However, the main change of shape occurs in the viscous sublayer, where the momentum flux is so low that a change in its contribution to the balance of forces in the control volume apparently makes little difference.

The main accomplishment of this work is the formulation of a method for computing the initial pressure rise in the nonseparated, supersonic, turbulent boundary layer interaction with a ramp. This also determines the boundary between the inner and outer layers and provides a rational starting point for calculating the interaction of the shock wave with the outer layer and the resulting pressure distribution. Thus, a complete and accurate solution for the pressure distribution over the first few boundary layer thicknesses downstream of the corner is obtained. The only inputs are the free-stream Mach number, the corner angle, and either the upstream skin friction coefficient or Reynolds number. This formulation is successful because it puts the complex part of the interaction into the control volume, which can be treated by an approximate (integral) method, while the simpler flow outside that region can be computed more exactly. It allows for the deep 
shock penetration, which actually occurs in these intexactions and for a realistic description of most of the supersonic portion of the boundary layex downstream of it. To obtain the shear stress after the corner and to describe the overall flow further downstream, where stresses become important, will require further effort, possibly building on the present model.

\section{References}

1. Rose, W. C., Murphy, J. D., and Watson, E. D., "Interaction of an Oblique Shock wave with a Turbulent Boundary Layer," AIAA Journal, Vol. 7, No. 12, 1969, pp. 2211-2221.

2. Roshko, A. and Thomke, G. J., "Supersonic, Turbulent Boundary-Layer Interaction With a Compression Corner at Very High Reynolds Number," Proceedings of the symposium on Viscous Interaction Phenomena in Supersonic Hypersonic Flow, USAF Aexospace Research Laboratories, WrightPatterson Air Force Base, Ohio, University of Dayton Press, May 1969, pp. 109-138.

3. Elfstrom, G. M., "Turbulent Hypersonic Flow at a Wedge-Compression Corner," JFM, Vo1. 53, Part 1, 1972, pp. 113-127.

4. Bradshaw, P., "An Improved Van Direst Skin-Fxiction Formula for Compressible Turbulent Boundary Layers," AIAA Journa1, Vol. 15, No. 2, February 1977, pp. 212-214.

5. Liepmann, H. W. and Roshko, A., Elements of Gas Dynamics, Wiley Press, 1957.

6. Settles, G. S., Fitzpatrick, T. J., and Bogdonoff, S. M., "A Detailed Study of Attached and Separated Compression Cornex Flowfields in High Reynolds Number Supersonic Flow," AIAA Paper 78-1167, July 1978.

7. Stewartson, K., "Multi-Structured Boundary Layers," Advances in Applied Mechanics, Vol. 14, Academic Press, New York, 1974, pp. 145-239.

8. Melnik, R. E. and Grossman, B., "Interactions of Normal Shock Waves With Turbulent Boundary Layers at Transonic Speeds," presented at the ONR Project SQUID Workshop on Transonic Flow Problems in Turbomachinery, U.S. Naval Postgraduate School, Monterey, California, 11-12 February 1976.

9. Inger, G. R. and Mason, W. H., "Analytical Theory of Transonic Normal Shock-Turbulent-Boundary Layer Interaction," A.IAA Journal, Vol. I4, September 1976, pp. 1266-1272. 
10. Messiter, A. F., "Boundary-Layer Separation," Proceedings of the Eighth U.S. National Congress of Applied Mechanics, University of California, Los Angeles, 26-30 June 1978.

11. Stratford, B. S., "The Prediction of Separation of the Turbulent Boundary Layer," JFM, Vol. 5, Part 1, 1959, pp. 1-16.

12. Hung, C. M. and MacCormack, R. W., "Numerical Simulation of Supersonic and Hypersonic Turbulent Compression Corner Flows," AIAA Journal, Vo1. 15, No. 3, March 1977, pp. 410-416.

13. Settles, G. S., Private Communication. 